\title{
THE CENTRAL SLOPE OF DARK MATTER CORES IN DWARF GALAXIES: SIMULATIONS VERSUS THINGS
}

\author{
Se-Heon Oh ${ }^{1,8}$, Chris Brook ${ }^{2}$, Fabio Governato $^{3}$, Elias Brinks ${ }^{4}$, Lucio Mayer $^{5}$, \\ W. J. G. DE BloK ${ }^{1}$, Alyson Brooks ${ }^{6}$, and Fabian Walter ${ }^{7}$ \\ ${ }^{1}$ Astronomy Department, Astrophysics, Cosmology, and Gravity Centre (ACGC), University of Cape Town, Private Bag X3, \\ Rondebosch 7701, South Africa; seheon_oh@ast.uct.ac.za, edeblok@ast.uct.ac.za \\ 2 Jeremiah Horrocks Institute, University of Central Lancashire, Preston, Lancashire, PR1 2HE, UK; cbbrook@uclan.ac.uk \\ ${ }^{3}$ Astronomy Department, University of Washington, Seattle, WA 98195, USA; fabio@astro.washington.edu \\ ${ }^{4}$ Centre for Astrophysics Research, University of Hertfordshire, College Lane, Hatfield, AL10 9AB, UK; E.Brinks@ @erts.ac.uk \\ ${ }^{5}$ Institute for Theoretical Physics, University of Zurich, Winterthurerstrasse 190, 8057 Zürich, Switzerland; lmayer@physik.unizh.ch \\ 6 Theoretical Astrophysics, California Institute of Technology, MC 350-17, Pasadena, CA 91125, USA; abrooks@ tapir.caltech.edu \\ ${ }^{7}$ Max-Planck-Institut für Astronomie, Königstuhl 17, 69117 Heidelberg, Germany; walter@mpia.de \\ Received 2010 November 5; accepted 2011 March 30; published 2011 June 9
}

\begin{abstract}
We make a direct comparison of the derived dark matter (DM) distributions between hydrodynamical simulations of dwarf galaxies assuming a $\Lambda$ CDM cosmology and the observed dwarf galaxies sample from the THINGS survey in terms of (1) the rotation curve shape and (2) the logarithmic inner density slope $\alpha$ of mass density profiles. The simulations, which include the effect of baryonic feedback processes, such as gas cooling, star formation, cosmic UV background heating, and most importantly, physically motivated gas outflows driven by supernovae, form bulgeless galaxies with DM cores. We show that the stellar and baryonic mass is similar to that inferred from photometric and kinematic methods for galaxies of similar circular velocity. Analyzing the simulations in exactly the same way as the observational sample allows us to address directly the so-called cusp/core problem in the $\Lambda \mathrm{CDM}$ model. We show that the rotation curves of the simulated dwarf galaxies rise less steeply than cold dark matter rotation curves and are consistent with those of the THINGS dwarf galaxies. The mean value of the logarithmic inner density slopes $\alpha$ of the simulated galaxies' DM density profiles is $\sim-0.4 \pm 0.1$, which shows good agreement with $\alpha=-0.29 \pm 0.07$ of the THINGS dwarf galaxies. The effect of non-circular motions is not significant enough to affect the results. This confirms that the baryonic feedback processes included in the simulations are efficiently able to make the initial cusps with $\alpha \sim-1.0$ to -1.5 predicted by DM-only simulations shallower and induce DM halos with a central mass distribution similar to that observed in nearby dwarf galaxies.
\end{abstract}

Key words: dark matter - galaxies: dwarf - galaxies: halos - galaxies: kinematics and dynamics

Online-only material: color figures

\section{INTRODUCTION}

The dark matter (DM) distributions at the centers of galaxies have been intensively discussed from both observational and theoretical perspectives for almost two decades ever since high-resolution $\mathrm{N}$-body DM simulations assuming a universe dominated by cold dark matter (CDM) and a cosmological constant $\Lambda$ were performed. The $\Lambda \mathrm{CDM}$ simulations have invariably predicted a DM density distribution that diverges toward the centers of galaxies (Moore 1994; Navarro et al. 1996, 1997, 2004; Moore et al. 1999b; Ghigna et al. 2000; Klypin et al. 2001; Power et al. 2002; Stoehr et al. 2003; Reed et al. 2005; Diemand et al. 2008). In order to describe such cusplike DM distributions, Navarro et al. $(1995,1996)$ proposed a profile (hereafter the NFW profile) that can be approximated by two power laws, $\rho \sim r^{-1.0}$ and $\rho \sim r^{-3.0}$, to describe the inner and outer regions of a DM halo, respectively. In particular, the central cusp feature with $\rho \sim r^{-1.0}$ has provided a useful test for $\Lambda \mathrm{CDM}$ cosmology and sparked interest in seeking constraints by observing mass distributions at the center of galaxies (e.g., McGaugh et al. 2003). It is worth mentioning that recent simulations show shallower slopes with $\alpha \sim-0.8$ at radii of $120 \mathrm{pc}$ (Stadel et al. 2009) although DM slopes at $r<1 \mathrm{kpc}$ are generally steeper (see also Navarro et al. 2010).

\footnotetext{
8 Square Kilometre Array South African Fellow.
}

The prediction of a central cusp from the $\Lambda$ CDM model has been seriously challenged by observations of dwarf and low surface brightness (LSB) disk galaxies. Observations of dwarf and LSB galaxies generally indicate a constant matter distribution toward their centers, with mass density profiles with a kiloparsec-sized core radius. This discrepancy of central DM distributions in dwarf galaxies in $\Lambda$ CDM simulations and observations is referred to as the "cusp/core" problem. This is a fundamental problem for $\Lambda \mathrm{CDM}$ together with the likely connected substructure and angular momentum problems (Moore et al. 1999a; Klypin et al. 1999; Simon \& Geha 2007; Navarro \& White 1994; Dutton 2009).

Compared to other types of galaxies, dwarf galaxies provide us with a good opportunity for measuring the DM distribution near the centers of galaxies due to the fact that they have a simple dynamical structure (disk galaxies without bulges) but also have low baryon fractions and hence less of a dynamical contribution by baryons (de Blok \& McGaugh 1997). Recently, Oh et al. (2011) presented high-resolution DM density profiles of seven dwarf galaxies taken from "The Hi Nearby Galaxy Survey" (THINGS; Walter et al. 2008). The high-quality data from THINGS significantly minimize observational uncertainties and thus allow us to investigate the central DM distribution of the dwarf galaxies in detail. Mass models of stars and gas are constructed using the Spitzer IRAC $3.6 \mu \mathrm{m}$ data from the "Spitzer Infrared Nearby Galaxies Survey" (SINGS; Kennicutt et al. 2003) and the total integrated THINGS H I map, 
respectively. The kinematics of baryons are then subtracted from the total kinematics of the galaxies in order to derive the DM distributions.

One of the main results of Oh et al. (2011) was to robustly confirm that the rotation curves of the seven THINGS dwarf galaxies rise too slowly to be consistent with a cusp feature at their centers. Moreover, the mean value of the logarithmic inner slopes of the DM density profiles is $\alpha=-0.29 \pm 0.07$, which significantly deviates from the $\alpha \sim-1.0$ predicted from DM-only simulations. The exquisite data used in this study allowed unprecedented treatment of the effects of observational uncertainties, such as beam smearing, center offset, and noncircular motions, which may play a role in hiding central cusps (Blais-Ouellette et al. 1999; van den Bosch et al. 2000; Bolatto et al. 2002; Swaters et al. 2003; Simon et al. 2003; Rhee et al. 2004; Gentile et al. 2005; Spekkens et al. 2005; Oh et al. 2008). The results have thus significantly strengthened the observational evidence that the DM distribution near the centers of dwarf galaxies follows a near-constant density core.

Before using these results as a repudiation of CDM, however, one must also examine carefully the modeling on which the central cusp predictions are based, which is usually done using $\mathrm{N}$-body simulations that include only the effects of gravity on structure formation. Although baryons make up only $\sim 14 \%$ of the matter of the universe, this dissipative constituent of the universe cools with cosmological time and accumulates in the central regions of DM halos, making up a dynamically important fraction. Several mechanisms have been proposed whereby these central baryons can affect the central cusp-like DM distribution that is found in pure DM simulations. A rapid change in potential (faster than the dynamical time) due to starburst-triggered outflows is one mechanism that has been shown to be capable of transforming cusp-like profiles into (flatter) cores (Navarro et al. 1996; Read \& Gilmore 2005; although see Gnedin \& Zhao 2002). Supernova (SN) driven random bulk motions of gas in protogalaxies (Mashchenko et al. 2006) has also been shown in models to flatten cusps, as have the effects of dynamical friction acting on gas clumps (El-Zant et al. 2002), and the transfer of angular momentum from baryons to the DM (Tonini et al. 2006). Modeling an inhomogeneous multiphase, interstellar medium (ISM) is critical for simulating the baryonic feedback processes in galaxies (Robertson \& Kravtsov 2008; Ceverino \& Klypin 2009). Yet cosmological simulations have not, until recently, been able to achieve enough resolution to model even such an inhomogeneous ISM, but have been forced to treat the important processes of star formation (SF) and feedback as "sub-grid" physics, averaging SF and SN feedback over large volumes, compared to the typical structural scales $(<1 \mathrm{kpc})$ of small galaxy disks.

Most recently, Governato et al. (2010) performed highresolution cosmological $\mathrm{N}$-body+smoothed particle hydrodynamic (SPH) simulations of dwarf galaxies under the $\Lambda \mathrm{CDM}$ paradigm that included the effect of baryonic feedback processes such as gas cooling, SF, cosmic UV background heating, and, most importantly, physically motivated gas outflows driven by SNe. The major finding of Governato et al. (2010) was that once $\mathrm{SF}$ is associated with high density gas regions, a significant amount of baryons with low angular momentum is efficiently removed by strong SNe-driven injection of thermal energy and the following gas outflows that carry an amount of gas at a rate of two to six times the local SF rate. The large-scale outflows in turn induce two effects. First, the loss of low angular momentum gas from the central regions prevents the formation of bulges in low-mass systems. Second, the clumpy nature of the gas and rapid ejection on short timescales has dynamical effects on the DM potential, creating a shallower density profile (see Mashchenko et al. 2006, 2008; Ceverino \& Klypin 2009; Mo \& Mao 2004).

Moreover, the simulated galaxies have a $z=0$ baryonic budget consistent with photometric and kinematic estimates (van den Bosch et al. 2001; McGaugh et al. 2010). The kinematic properties of the simulated dwarf galaxies are very similar to those of the THINGS dwarf galaxies in terms of their maximum rotation velocity $\left(\sim 60 \mathrm{~km} \mathrm{~s}^{-1}\right)$ and dynamical mass $\left(\sim 10^{9} M_{\odot}\right)$, allowing a direct comparison between the simulations and observations to be made.

A first estimate of the bulge-to-disk ratio and DM properties of these models was presented in Governato et al. (2010) by fitting a Sérsic profile to artificial $i$-band images and fitting a rotation curve to the rotational motions of cold gas using a tiltedring analysis. Here, we make more direct comparisons with observations, applying the same analysis techniques and tools to the simulations as done in the most recent observational sample of analogous galaxies. The determination of the DM slopes for the simulations has to be done in the same way as in observations since there are various aspects that can affect the answer, such as beam smearing, center offset, and non-circular motions, which were not taken into account when the slope was derived from the raw simulation data in Governato et al. (2010). This approach will have two crucial benefits: (1) provide a strong test of theoretical predictions and (2) test the extent of observational biases possibly associated with current models of baryon/ DM mass decompositions in real galaxies, in particular the recovery of non-circular motions and pressure support induced by SN feedback (Valenzuela et al. 2007; Dalcanton \& Stilp 2010).

The structure of this paper is as follows. The simulations are described in Section 2. In Section 3, we present the DM mass modeling of the simulated dwarf galaxies. Section 4 compares the derived DM distributions from the simulations with those of the THINGS dwarf galaxies. Finally, we summarize the main results and conclusions of this paper in Section 5.

\section{THE SPH TREECODE GASOLINE}

The halos for these simulations were selected from a set of large-scale, low-resolution, DM-only simulation run in a concordance, flat, $\Lambda$-dominated cosmology: $\Omega_{0}=0.24, \Lambda=$ $0.76, h=0.73, \sigma_{8}=0.77$, and $\Omega_{b}=0.042$ (Verde et al. 2003). The size of the box, $25 \mathrm{Mpc}$, is large enough to provide realistic torques for the small galaxies used in this work. The power spectra to model the initial linear density field were calculated using the CMBFAST code to generate transfer functions. To include the effects of cosmic torques from the large-scale structure, we used the volume renormalization (or "zoom in") technique (Katz \& White 1993). DM particle masses in the high-resolution regions are $1.6 \times 10^{4} M_{\odot}$, while the mass of star particles is only $1000 M_{\odot}$ and the force resolution, i.e., the gravitational softening, is $86 \mathrm{pc}$. In total, at $z=0$ there are $3.3 \times 10^{6}$ particles within the virial radius of the simulated dwarf galaxy, hereafter referred to as DG1. For all particle species, the gravitational spline softening, $\epsilon(z)$, was evolved in a comoving manner from the starting redshift $(z \sim 100)$ until $z=8$ and then remained fixed at its final value from $z=8$ to the present. At $z=0$ the virial masses of the halos that we studied in this paper are $3.5(\mathrm{DG} 1)$ and $2.0(\mathrm{DG} 2) \times 10^{10} M_{\odot}$ (the virial mass is 


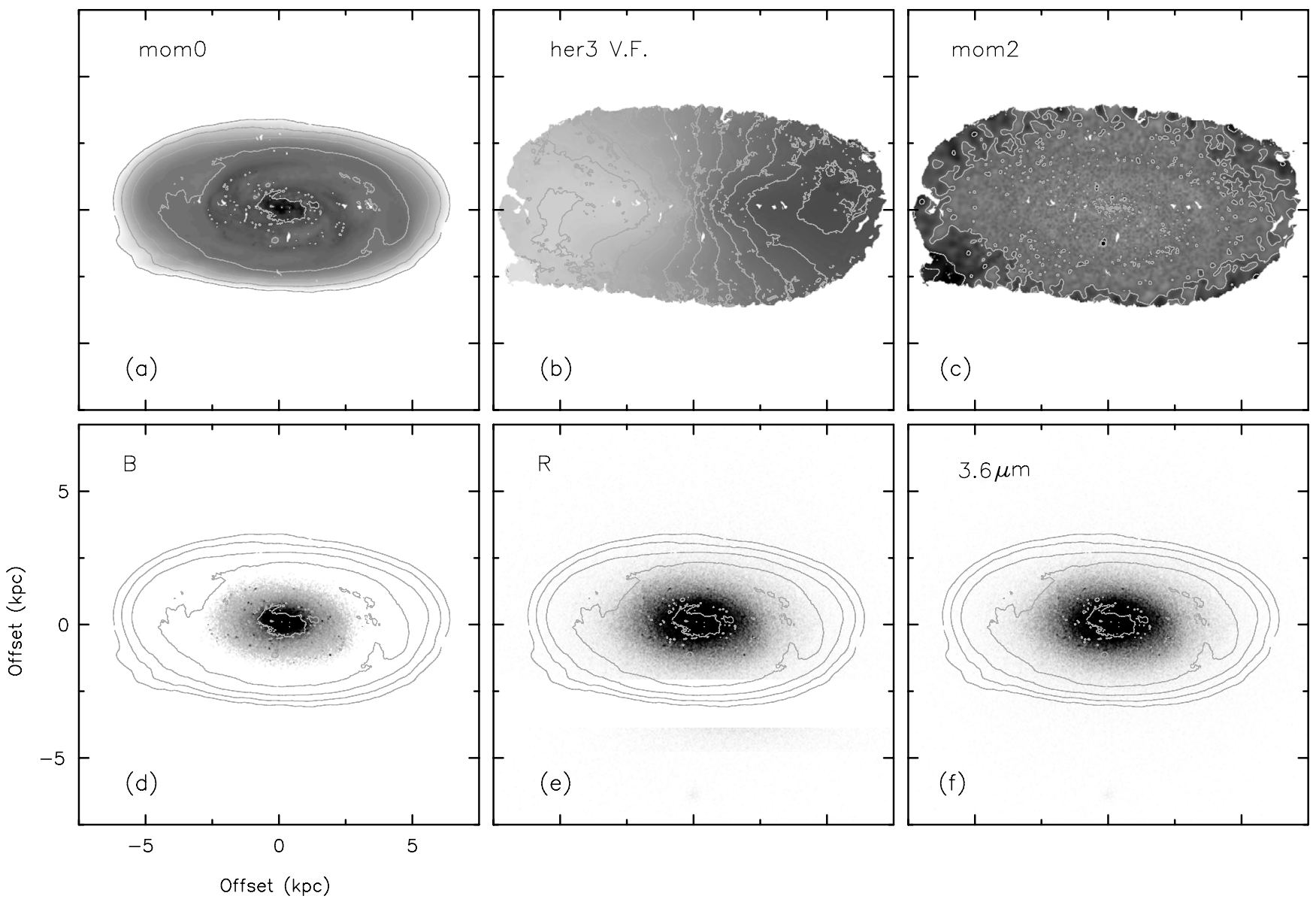

Figure 1. Gas and stellar components of DG1. (a) Simulated integrated gas map (moment 0). Contours start from a column density of $10^{20} \mathrm{~cm}^{-2}$ to $10^{22} \mathrm{~cm}^{-2}$ in steps of $10^{0.4} \mathrm{~cm}^{-2}$. (b) Hermite $h_{3}$ velocity field. Velocity contours run from $-50 \mathrm{~km} \mathrm{~s}^{-1}$ to $50 \mathrm{~km} \mathrm{~s}^{-1}$ with a spacing of $10 \mathrm{~km} \mathrm{~s}$. (c) Velocity dispersion map (moment 2). Velocity contours run from $5 \mathrm{~km} \mathrm{~s}^{-1}$ (gray) to $15 \mathrm{~km} \mathrm{~s}^{-1}$ (black) with a spacing of $4 \mathrm{~km} \mathrm{~s}^{-1}$. (d)-(f) Total intensity maps in simulated optical $B, R$, and Spitzer IRAC $3.6 \mu \mathrm{m}$ bands with superimposed contours of the gas map in panel (a).

measured within the virial radius $R_{\mathrm{vir}}$, the radius enclosing an overdensity of 100 times the cosmological critical density).

To evolve the simulations described here we have used the fully parallel, $N$-body+SPH code GASOLINE to compute the evolution of both the collisionless and dissipative component in the simulations. A detailed description of the code is available in the literature (Wadsley et al. 2004). The version of the code used in this paper includes radiative cooling and accounts for the effect of a uniform background radiation field on the ionization and excitation state of the gas. The cosmic ultraviolet background is implemented using the Haardt-Madau model (Haardt \& Madau 1996), including photoionizing and photoheating rates produced by Pop III stars, QSOs, and galaxies starting at $z=9$. We use a standard cooling function for a primordial mixture of atomic hydrogen and helium at high gas temperatures and we include low temperature cooling (Mashchenko et al. 2006).

In the simulations described in this paper, SF occurs when cold gas reaches a given threshold density (e.g., Stinson et al. 2006) typical of actual star-forming regions (we used 100 atomic mass unit $\left.(\mathrm{amu}) \mathrm{cm}^{-3}\right)$. SF then proceeds at a rate proportional to $\rho_{\text {gas }}^{1.5}$, i.e., locally enforcing a Schmidt law. The adopted feedback scheme is implemented by releasing thermal energy from $\mathrm{SNe}$ into the gas surrounding each star particle (Stinson et al. 2006). The energy release rate is tied to the time of formation of each particle (which effectively ages as a single stellar population with a Kroupa initial mass function). To model the effect of feedback at unresolved scales, the affected gas has its cooling shut off for a timescale proportional to the Sedov solution of the blast wave equation, which is set by the local density and temperature of the gas and the amount of energy involved. The effect of feedback is to regulate SF in the disks of massive galaxies and to greatly lower the SF efficiency in galaxies with peak circular velocity in the $50 \mathrm{~km} \mathrm{~s}^{-1}<S V_{c}<150 \mathrm{~km} \mathrm{~s}^{-1}$ range (Brooks et al. 2007). At even smaller halo masses $\left(V_{c}<\right.$ $20-40 \mathrm{~km} \mathrm{~s}^{-1}$ ), the collapse of baryons is largely suppressed by the cosmic UV field (Quinn et al. 1996; Okamoto et al. 2008; Gnedin 2010).

Other than the density threshold only two other parameters are needed, the SF efficiency $(\epsilon \mathrm{SF}=0.1)$ and the fraction of SN energy coupled to the ISM $(\epsilon \mathrm{SN}=0.4)$. The model galaxies studied in this paper are those published in Governato et al. (2010). We verified that the addition of full metal cooling and the increase of $\epsilon \mathrm{SN}$ to 1 does not substantially change the structural properties of the galaxy. However, the amount of stars formed decreases by $40 \%$.

As a benchmark against which the effects of baryonic feedback processes can be gauged, Governato et al. (2010) performed additional runs for analogous model galaxies of DG1, called DG1DM and DG1LT. DG1DM uses the same initial conditions as DG1 but includes only the DM component. It has a DM slope of $\alpha \sim-1.3$ similar to those found from similar simulations (Springel et al. 2008). DG1LT is a version of DG1 using a lower density threshold $\left(0.1 \mathrm{amu} \mathrm{cm}^{-3}\right)$ at a 
lower resolution (the force resolution is $\sim 116 \mathrm{pc}$ ), and outflows in this model are negligible. DG1LT has a cusp and its DM slope is similar to that of DG1DM $(\alpha \sim-1.3)$.

To properly compare the outputs from the simulation to real galaxies and make accurate estimates of the observable properties of galaxies (e.g., Sánchez-Janssen et al. 2010), we used the Monte Carlo radiation transfer code suNRISE (Jonsson et al. 2010) to generate artificial optical images and spectral energy distributions (SEDs) of the outputs of our run. SUNRISE allows us to measure the dust-reprocessed SED of every resolution element of the simulated galaxies, from the far-UV to the far-IR, with a full three-dimensional treatment of radiative transfer. We place the simulated galaxies at an inclination of $60^{\circ}$. However, in applying our analysis tools, the inclination is considered a free parameter, in keeping with the techniques applied to observed galaxies. Filters mimicking those of the Sloan Digital Sky Survey (SDSS) are used to create mock observations.

We note that in the runs adopting the "high threshold" SF, feedback produces winds that are comparable in strength to those found in real galaxies of similar mass. However, in our simulations the cold ISM is still only moderately turbulent $\left(\sim 5-10 \mathrm{~km} \mathrm{~s}^{-1}\right.$ at $\left.z=0\right)$, consistent with observations, and the galaxies match the observed stellar and baryonic Tully-Fisher relation (Governato et al. 2009), as the SF efficiency is regulated to form an amount of stars similar to that of real dwarf galaxies of similar rotation velocity.

In the following sections, we perform DM mass modeling of both DG1 and DG2 in exactly the same way as the THINGS dwarf galaxies sample described in Oh et al. (2008, 2011).

\section{THE MASS MODELING OF THE SIMULATED DWARF GALAXIES}

\subsection{The Rotation Curves}

We first construct the data cubes of DG1 and DG2 by tracing the motions of the gas component. The beam and velocity resolutions of the cubes are $\sim 6^{\prime \prime}$ (corresponding to $100 \mathrm{pc}$ at a distance of $4 \mathrm{Mpc}$ ) and $2.0 \mathrm{~km} \mathrm{~s}^{-1}$, respectively. As an example, we show the integrated gas map, velocity field, and velocity dispersion map extracted from the cube for DG1 in Figure 1. For the velocity field, we use the Gauss-Hermite polynomial to model the skewness of a non-Gaussian profile caused by multiple velocity components (van der Marel \& Franx 1993). This function includes an extra parameter, called $h_{3}$, that measures the skewness of the Gaussian function and thus provides more reliable central velocities even for profiles with significant asymmetries. As discussed in Oh et al. (2011) (see also de Blok et al. 2008), the hermite $h_{3}$ velocity field gives a robust estimate for the underlying circular rotation of a galaxy in which non-circular motions are insignificant, like DG1 and DG2. Hermite $h_{3}$ polynomials have also been used to extract the velocity fields of the THINGS galaxies sample (de Blok et al. 2008).

Oh et al. (2011) use the bulk velocity fields when deriving the rotation curves of the THINGS dwarf galaxies, except for DDO 154 and M81dwB in which non-circular motions are insignificant. Compared to other types of velocity fields (e.g., intensity-weighted mean, hermite $h_{3}$, single Gaussian, and peak velocity fields), the bulk velocity field more effectively minimizes the effect of small-scale random motions on the derived kinematics of a galaxy (see Oh et al. 2008 for details).
However, for a galaxy that is not significantly affected by noncircular motions like DG1 and DG2, the bulk velocity field is nearly identical to the hermite $h_{3}$ velocity field.

In Figure 1(b), the iso-velocity contours of the extracted hermite $h_{3}$ velocity field are distorted in some regions, suggestive of non-circular motions. These are mainly due to the SN-driven gas outflows in the simulations. However, despite the presence of non-circular motions the overall pattern of the galaxy rotation is well recovered in the velocity field.

It is customary to use a set of concentric tilted rings to model the velocity field of a galaxy, each with its own kinematic center (XPOS, YPOS), inclination INCL, position angle P.A., expansion velocity VEXP, systemic velocity VSYS, and rotation velocity VROT (Begeman 1989). P.A. is the angle measured counterclockwise from the north direction in the sky to the major axis of the receding half of the galaxy.

Assuming an infinitely thin disk, we fit these tilted rings to the hermite $h_{3}$ velocity fields of DG1 and DG2 to derive their rotation curves. The derived tilted-ring models for DG1 and DG2 are shown in Figure 2. The error bar in the rotation velocities indicates the dispersion of individual velocity values found along a tilted ring. As mentioned earlier, gas outflows driven by $\mathrm{SN}$ explosions in the simulations cause non-circular motions at some regions and induce larger uncertainties in the fitted tilted rings. These are seen as the scatter of the very first run results (open circles) with all ring parameters free in Figure 2. However, the local scatter averages out after several iterations and the final rotation curves (solid lines) in Figure 2 seem to give a good description of the underlying kinematics of DG1 and DG2. As shown in the VROT panels of Figure 2, this can be confirmed by the true rotation velocities (dotted lines) derived using the full three-dimensional mass distributions rather than the projected two-dimensional surface density profiles of the simulated galaxies. Despite not only the uncertainties but also the assumption of the tilted-ring analysis, i.e., an infinitely thin disk, the difference between the true and derived rotation velocities is less than $5 \mathrm{~km} \mathrm{~s}^{-1}$, equivalent to about twice the velocity resolution of the cubes.

For galaxies whose velocity dispersions are large enough compared to their maximum rotation velocities, we need to correct for the asymmetric drift to obtain more reliable rotation velocities (Bureau \& Carignan 2002). However, the second moment maps of DG1 and DG2 show small velocity dispersions $\left(\sim 7 \mathrm{~km} \mathrm{~s}^{-1}\right)$ compared to the maximum rotation velocities $\left(\sim 60 \mathrm{~km} \mathrm{~s}^{-1}\right)$, and the pressure support is insignificant with respect to the circular rotations. Therefore, we ignore the asymmetric drift corrections for DG1 and DG2.

\subsection{The Mass Models of Baryons}

The derived rotation curves in the previous section represent the total kinematics of the galaxies, including not only DM but also the stellar and gas components. We therefore construct mass models of the baryons and subtract them from the total kinematics to separate the DM component only.

For the gas component, we first derive the gas surface density profile by applying the derived tilted rings in Section 3.1 to the integrated gas map shown in the upper left panel of Figure 1 . The derived gas surface density profile is then scaled by a factor of 1.4 to account for helium and metals. The resulting gas surface density profiles of DG1 and DG2 are given in panels (e) of Figure 3. From these, we calculate the rotation velocity due to the gas component, assuming an infinitely thin disk. The derived gas rotation velocities of DG1 and DG2 are 

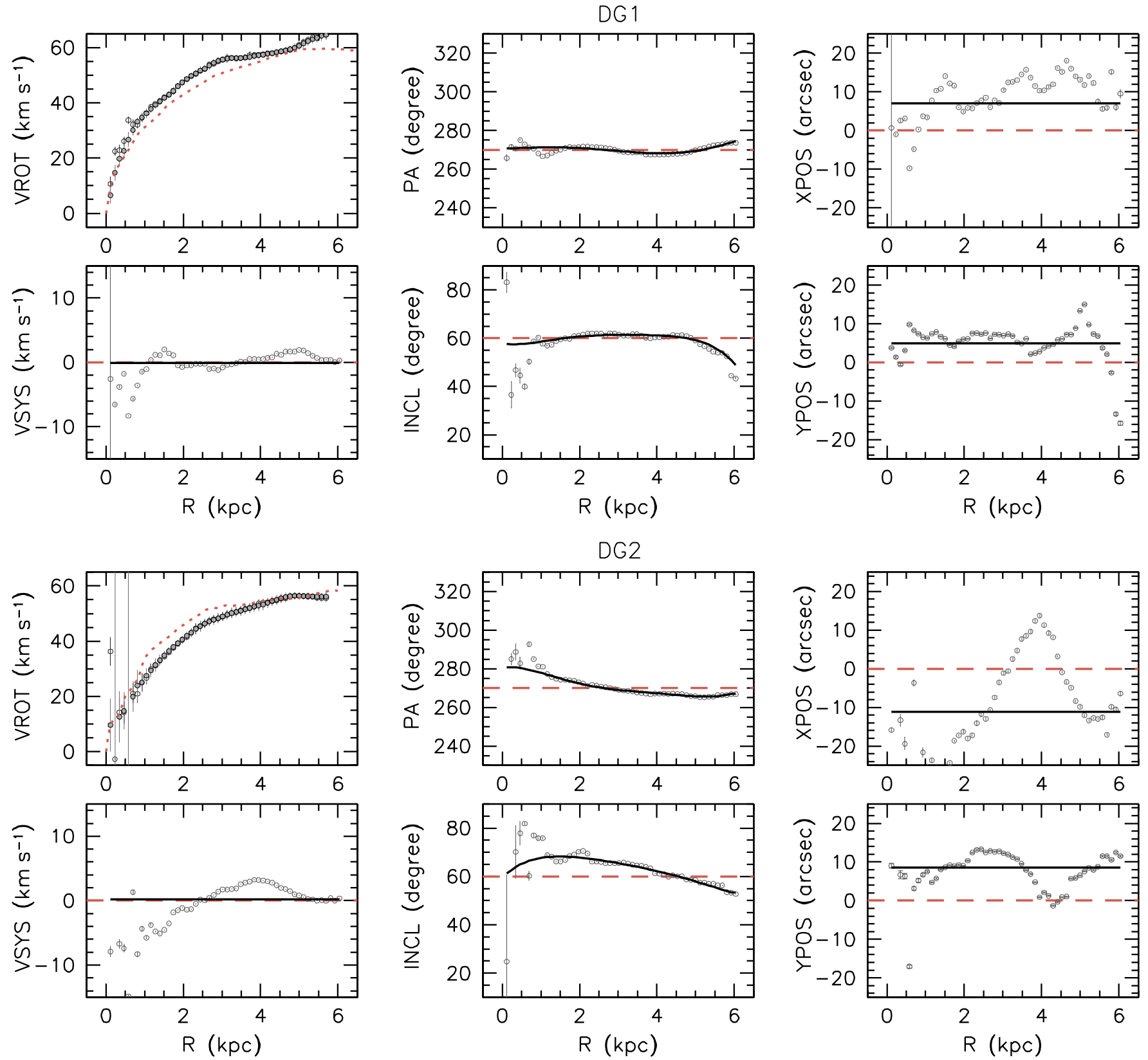

Figure 2. Tilted-ring models derived from the hermite $h_{3}$ velocity fields of DG1 (upper) and DG2 (lower). The open circles in all panels indicate the fit made with all parameters free. The filled black circles and solid lines in all panels show the finally adopted tilted-ring models as a function of galaxy radius. The dashed lines indicate the geometrical parameters used when extracting the rotation velocities (dotted lines in the VROT panels) from the true mass distributions of the simulated galaxies.

(A color version of this figure is available in the online journal.)

shown in panels (f) of Figure 3. We also overplot the true values derived using the full three-dimensional mass distribution of the gas components of DG1 and DG2 as shown in the open circles in panels (e) of Figure 3. For DG1, the true and derived gas surface density profiles are similar but the derived rotation velocity is systematically higher than the true one. This can be due to the assumption of "an infinitely thin disk" which makes one overestimate the gas rotation velocity of a galaxy with a considerable gas thickness. However, the velocity difference $\left(\sim 5 \mathrm{~km} \mathrm{~s}^{-1}\right)$ is not very significant. For DG2, the true

\footnotetext{
9 The gas rotation velocities assuming "an exponential density law" with scale heights in the range of $0.5-2.5 \mathrm{kpc}$ are somewhat similar to the true one but slightly higher in the outer regions, possibly due to $\mathrm{SNe}$-driven gas outflows or flaring.
}

gas velocity is systematically higher in the range $0-3 \mathrm{kpc}$, particularly in the inner region. Likewise, the true surface density profile is higher than the derived one in the range $0-3 \mathrm{kpc}$. As shown in Figure 2, this is partially because the smaller inclination value $\left(60^{\circ}\right)$ is used for extracting the true gas surface density profile from the simulation in the inner region of DG2. In addition, this may also be due to significant vertical gas outflows or flaring driven by $\mathrm{SNe}$ perpendicular to the disk of the galaxy.

Similar to the gas component, we derive the surface density profiles for the stellar components of DG1 and DG2. For this, as shown in Figure 1, we use the simulated $B, V, R$, and Spitzer IRAC $3.6 \mu \mathrm{m}$ images. In particular, the Spitzer IRAC $3.6 \mu \mathrm{m}$ image is useful for tracing the underlying old stars which are 

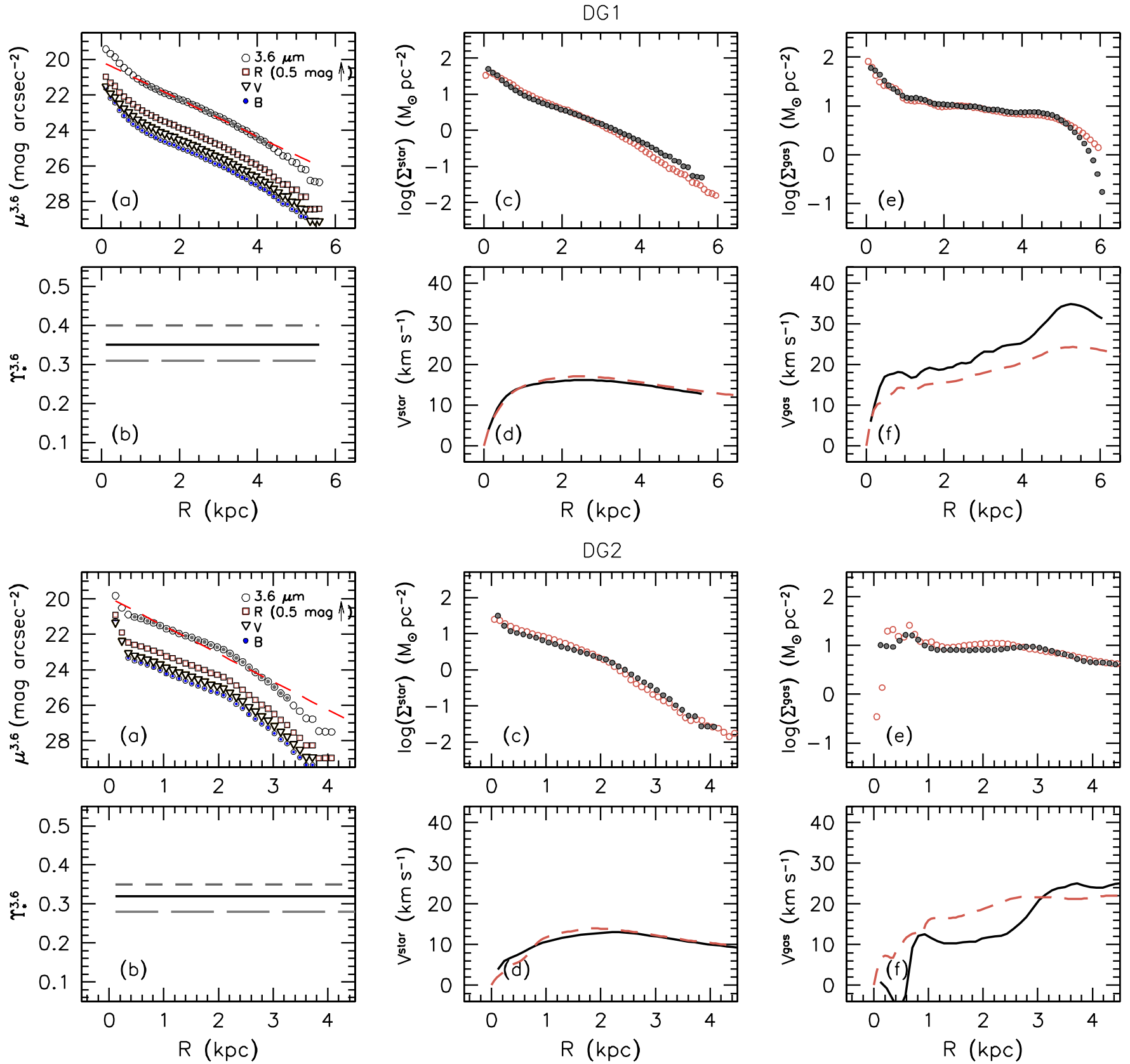

Figure 3. Mass models for the baryons of DG1 (top) and DG2 (bottom). (a) Azimuthally averaged surface brightness profiles (corrected for inclination) in the simulated $3.6 \mu \mathrm{m}, R, V$, and $B$ bands (top to bottom) derived applying the tilted-ring parameters shown in Figure 2. Note that the $R$-band surface brightness profile is shifted upward by $0.5 \mathrm{mag}$ for clarity. The dashed line for the $3.6 \mu \mathrm{m}$ profile indicates a least-squares fit to the data, the radial range over which the fit is made being indicated by the filled circles. (b) The $\Upsilon_{\star}$ in the $3.6 \mu \mathrm{m}$ band derived from stellar population synthesis models. The short and long dashed lines show the $\Upsilon_{\star}^{3.6}$ values derived using optical colors $B-V$ and $B-R$, respectively. The solid line indicates the mean value adopted as the final $\Upsilon_{\star}^{3.6}$. (c) The stellar mass surface density derived from the $3.6 \mu \mathrm{m}$ surface brightness in (a) using the $\Upsilon_{\star}^{3.6}$ value shown in panel (b). The red open circles indicate the true profile derived from the simulations. (d) The rotation velocity for the stellar component derived from the stellar mass density profile (dots) in panel (c). The dashed line shows the true rotation velocity for the stellar component derived from the true profile (open circles) shown in panel (c). (e) The radial mass surface density distribution of the gas component scaled by 1.4 to account for He and metals. The red open circles indicate the true profile derived from the simulations. (f) The gas rotation velocity derived from the gas surface density profile (dots) in panel (e). The dashed line shows the true rotation velocity for the gas component derived from the true profile (open circles) shown in panel (e).

(A color version of this figure is available in the online journal.)

usually the dominant stellar population in dwarf galaxies. For the same reason, Spitzer IRAC $3.6 \mu \mathrm{m}$ images have also been used for making mass models for the stellar components of the THINGS galaxies sample (de Blok et al. 2008; Oh et al. 2008, 2011).

We derive the surface brightness profiles of DG1 and DG2 by applying the tilted rings derived in Section 3.1 to their simulated $B, V$, and $R$ as well as $3.6 \mu \mathrm{m}$ images. Both DG1 and DG2 are bulgeless as found from fitting a Sérsic profile to their $i$-band images (Governato et al. 2010). To convert the surface brightness profiles to the mass density profiles in units of $M_{\odot} \mathrm{pc}^{-2}$, we obtain the $3.6 \mu \mathrm{m}$ mass-to-light $\left(\Upsilon_{\star}^{3.6}\right)$ values using an empirical relation between $\Upsilon_{\star}^{3.6}$ and optical colors based on the Bruzual \& Charlot (2003) stellar population synthesis models (Oh et al. 2008; see also Bell \& de Jong 2001). The $\Upsilon_{\star}^{3.6}$ values used for DG1 and DG2 are shown as the solid lines in panels (b) of 

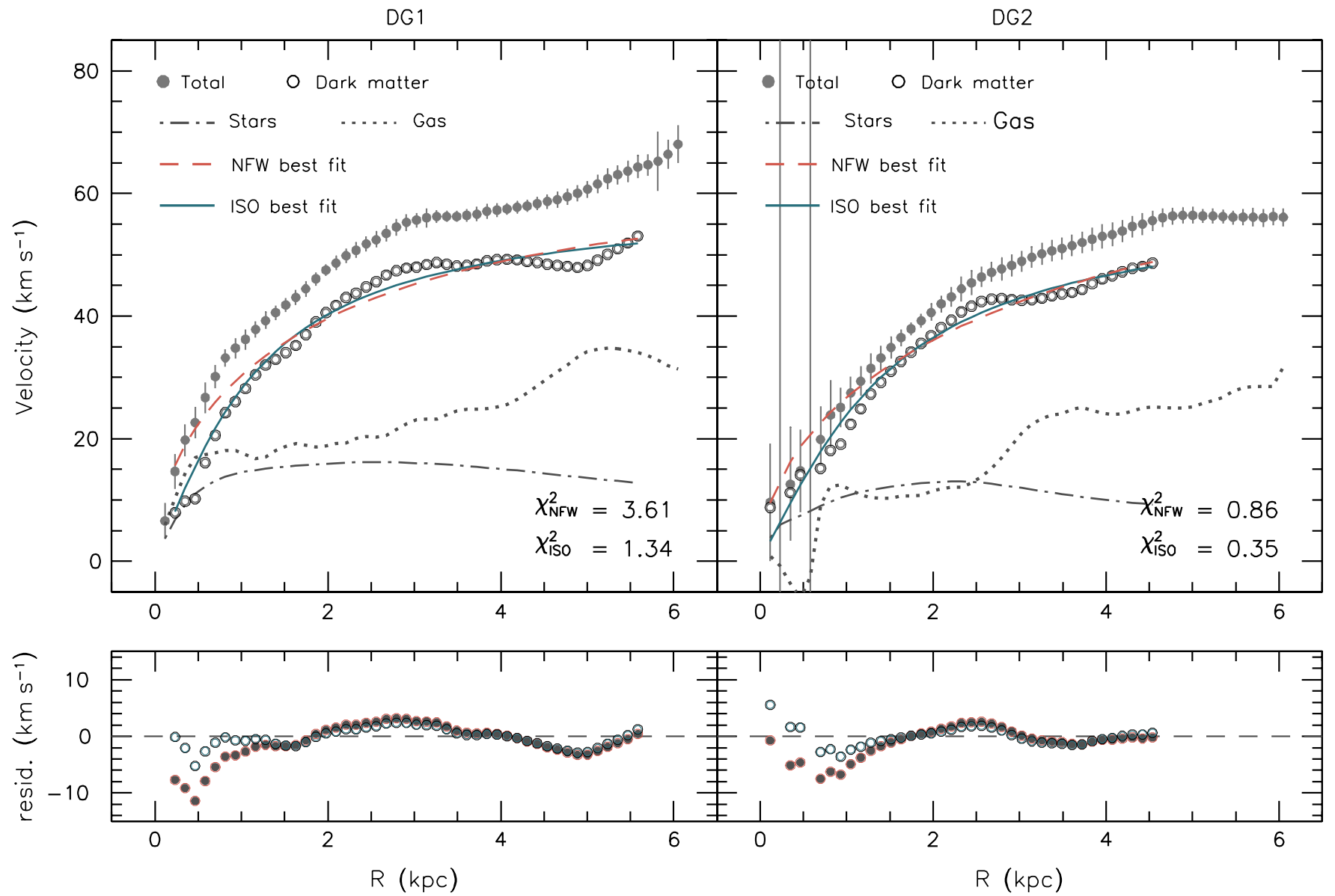

Figure 4. Disk-halo decompositions of the rotation curves of DG1 (left) and DG2 (right). The gray dots represent the total rotation curves derived from the hermite $h_{3}$ velocity fields. The dotted and dash-dotted lines indicate the rotation curves of the gas and stellar components, respectively. The open circles show the DM rotation curves derived subtracting the rotation curves of baryons from the total rotation curves. The dashed and solid lines show the best-fitted NFW and ISO halo models to the DM rotation curves, respectively. The reduced $\chi^{2}$ value for each halo model is denoted on the panels. The lower panels show the velocity residuals between the DM rotation curves and the best-fitted halo models. The dots and open circles indicate the results from the NFW and ISO halo models, respectively.

(A color version of this figure is available in the online journal.)

Table 1

Properties of the Simulated and the THINGS Dwarf Galaxies

\begin{tabular}{|c|c|c|c|c|c|c|c|c|c|c|}
\hline Name & $\begin{array}{c}D \\
(\mathrm{Mpc}) \\
(1) \\
\end{array}$ & $\begin{array}{c}\langle\text { Incl. }\rangle \\
\left({ }^{\circ}\right) \\
(2) \\
\end{array}$ & $\begin{array}{c}z_{0} \\
(\mathrm{kpc}) \\
(3) \\
\end{array}$ & $\begin{array}{c}M_{\mathrm{B}} \\
(\mathrm{mag}) \\
(4) \\
\end{array}$ & $\begin{array}{c}V_{\max } \\
\left(\mathrm{km} \mathrm{s}^{-1}\right) \\
(5)\end{array}$ & $\begin{array}{c}R_{\max } \\
(\mathrm{kpc}) \\
(6) \\
\end{array}$ & $\begin{array}{c}M_{\mathrm{dyn}} \\
\left(10^{9} M_{\odot}\right) \\
(7) \\
\end{array}$ & $\begin{array}{c}M_{\text {halo }} \\
\left(10^{9} M_{\odot}\right) \\
(8) \\
\end{array}$ & $\begin{array}{c}M_{\text {star }} \\
\left(10^{8} M_{\odot}\right) \\
(9) \\
\end{array}$ & $\begin{array}{c}M_{\mathrm{gas}} \\
\left(10^{8} M_{\odot}\right) \\
(10) \\
\end{array}$ \\
\hline IC 2574 & 4.0 & 55.7 & 0.57 & -18.1 & 77.6 & 10.4 & 14.6 & 53.2 & 10.38 & 18.63 \\
\hline Holmberg I & 3.8 & 13.9 & 0.55 & -14.8 & 38.0 & 1.5 & 0.5 & 33.1 & 1.25 & 2.06 \\
\hline Holmberg II & 3.4 & 49.6 & 0.28 & -16.9 & 35.5 & 7.1 & 2.1 & 4.3 & 2.00 & 7.41 \\
\hline M81 dwB & 5.3 & 44.8 & 0.09 & -14.2 & 39.8 & 0.8 & 0.3 & 870.9 & 0.30 & 0.31 \\
\hline$\overline{\text { DG1 }}$ & 4.0 & 60.0 & 0.41 & -15.9 & 60.0 & 5.0 & 4.2 & 35.0 & 1.81 & 8.04 \\
\hline DG2 & 4.0 & 60.0 & 0.28 & -15.6 & 56.4 & 5.0 & 3.7 & 20.0 & 0.80 & 5.92 \\
\hline
\end{tabular}

Notes. (1) Distance as given in Walter et al. (2008). For DG1 and DG2, we assume they are at a distance of 4 Mpc. (2) Average value of the inclination derived from the tilted-ring analysis. (3) The vertical scale height of disk. (4) Absolute $B$ magnitude as given in Walter et al. (2008). (5) Maximum rotation velocity. (6) The radius where the rotation velocity $V_{\max }$ of the flat part of the rotation curve is measured. (7) Dynamical mass from the measured $V_{\max }$ and $R_{\max }$. (8) Halo mass $M_{200}$ determined from $V_{200}$ using Equation (3). For the THINGS dwarf galaxies, we use the $V_{200}$ values fitted using $c$ fixed to 5 . See Section 4.1 for more details. For DG1 and DG2, we show the virial mass measured within the virial radius $R_{\text {vir }}$ enclosing an overdensity of 100 times the cosmological critical density. (9) Stellar mass derived in Section 3.2. The stellar mass of DDO 154 is from de Blok et al. (2008). (10) Gas mass derived in Section 3.2. The gas mass of DDO 154 is from Walter et al. (2008).

Figure 3, and the resulting stellar surface density profiles are given in panels (c) of Figure 3. The true surface density profiles for the stellar components of DG1 and DG2 are also overplotted as the open circles in Figure 3, and they are similar to the derived ones. This can be treated as circumstantial evidence that the assumption used for the stellar distribution and $\Upsilon_{\star}^{3.6}$ values 
provide a good description for the stellar components of DG1 and DG2.

From these, we then compute the corresponding stellar rotation velocities assuming a vertical $\operatorname{sech}^{2}(z)$ scale height distribution of stars. We calculate the vertical scale height $z_{0}$ using a ratio of $h / z_{0}=2.5$, where $h$ is the radial scale length of disk, derived from the $3.6 \mu \mathrm{m}$ surface brightness profile. The derived scale heights $z_{0}$ of DG1 and DG2 are $0.41 \mathrm{kpc}$ and $0.28 \mathrm{kpc}$, respectively. These are similar to the mean value $(0.32 \mathrm{kpc})$ of the seven THINGS dwarf galaxies as given in $\mathrm{Oh}$ et al. (2011). The derived stellar rotation velocities of DG1 and DG2 are shown in panels (d) of Figure 3, and they agree well with the true ones indicated by dashed lines.

\subsection{The Disk-Halo Decomposition}

We separate the DM components of DG1 and DG2 by subtracting the mass models of baryons derived in Section 3.2 from their total rotation curves. We then fit two halo models, the NFW and pseudo-isothermal (ISO) halo models, to these kinematic residuals in order to examine the DM distribution in a quantitative way. The NFW and ISO halo models represent cusp-like and constant density (core) matter distributions at the centers of galaxies, respectively.

The NFW halo model (Navarro et al. 1996, 1997) is given as

$$
V_{\mathrm{NFW}}(R)=V_{200} \sqrt{\frac{\ln (1+c x)-c x /(1+c x)}{x[\ln (1+c)-c /(1+c)]}},
$$

where $c$ is the parameter quantifying the degree of concentration of the DM halo. $V_{200}$ is the rotation velocity at radius $R_{200}$ where the density contrast with the critical density of the universe exceeds 200 and $x$ is defined as $R / R_{200}$.

Likewise, the rotation velocity based on the ISO halo model is as follows:

$$
V_{\mathrm{ISO}}(R)=\sqrt{4 \pi G \rho_{0} R_{C}^{2}\left[1-\frac{R_{C}}{R} \operatorname{atan}\left(\frac{R}{R_{C}}\right)\right]},
$$

where $\rho_{0}$ and $R_{C}$ are the core density and core radius of a halo, respectively.

By comparing the fit qualities of these two halo models to the kinematic residuals for the DM component, we examine which halo model is preferred to describe the derived DM distributions of DG1 and DG2.

As shown in the upper panels of Figure 4, compared to the CDM NFW halo model, despite its feasible fits, the ISO halo model gives a better description for the derived DM distributions of both DG1 and DG2 in terms of the fit quality (i.e., reduced $\chi^{2}$ values). This is also confirmed by the velocity residuals between the DM rotation curves and the best-fitted halo models as shown in the lower panels of Figure 4. The best-fitted NFW halo models are too steep to match the inner regions of the DM rotation curves of DG1 and DG2.

\section{THE SIMULATIONS VERSUS THINGS}

In this section, we compare the derived DM distribution of DG1 and DG2 with that of the seven THINGS dwarf galaxies. THINGS is a high spectral $\left(\leqslant 5.2 \mathrm{~km} \mathrm{~s}^{-1}\right)$ and angular $\left(\sim 6^{\prime \prime}\right)$ resolution $\mathrm{H}_{\mathrm{I}}$ survey for 34 nearby galaxies undertaken using the NRAO ${ }^{10}$ Very Large Array (Walter et al. 2008). THINGS is

\footnotetext{
10 The National Radio Astronomy Observatory is a facility of the National Science Foundation operated under cooperative agreement by Associated Universities, Inc.
}

complemented with other data, such as from the Spitzer SINGS survey and ancillary optical $B, V$, and $R$ images taken with the KPNO $2.1 \mathrm{~m}$ telescope (Kennicutt et al. 2003). These highquality multi-wavelength data significantly reduce observational uncertainties and thus enable us to derive more reliable mass models of the galaxies.

Oh et al. (2011) performed DM mass modeling of seven dwarf galaxies in exactly the same way as for DG1 and DG2 as described in Section 3. Basic properties of the galaxies are listed in Table 1. In particular, the selected seven THINGS dwarf galaxies have similar observational properties as DG1 and DG2, such as resolution $(\sim 150 \mathrm{pc}$ at the distance of $\sim 4 \mathrm{Mpc})$, maximum rotation velocity $\left(<80 \mathrm{~km} \mathrm{~s}^{-1}\right)$, dynamical mass $\left(\sim 10^{9} M_{\odot}\right)$, and scale height $(\sim 0.3 \mathrm{kpc})$. In addition, the THINGS dwarf galaxies are not satellites and they have most likely only weakly interacted with larger systems, as is the case for DG1 and DG2. Therefore, this allows us to make a direct comparison between the simulations and observations and examine if the simulated dwarf galaxies are realistic compared to the dwarf galaxies in the local universe.

\subsection{The Relation between $M_{\text {star }}$ and $M_{\text {halo }}$}

By comparing the stellar masses of DG1 and DG2 to their halo masses, we examine whether the SF efficiency of DG1 and DG2 is comparable with that of real galaxies that have similar dynamical masses. This is important as the number of stars that form in small halos can put strong constraints on baryonic feedback and its effects.

It has been found that galaxies of smaller or larger halo masses with respect to the Milky Way appear to have inefficient SF (Navarro \& Steinmetz 2000; Governato et al. 2007; Li \& White 2009). The low SF efficiency in high-mass galaxies is often attributed to active galactic nucleus feedback (Ciotti \& Ostriker 2001; Benson \& Madau 2003), while the effects of $\mathrm{SNe}$ are often invoked to explain the low SF efficiency in lowmass galaxies (Larson 1974; White \& Rees 1978). Recently, Guo et al. (2010) derived the relation between halo mass and stellar mass from abundance matching by combining the stellar mass function from the SDSS Data Release 7 (SDSS-DR7; Li \& White 2009) with the halo/subhalo mass function from $N$-body $\Lambda \mathrm{CDM}$ simulations (Millennium and Millennium-II simulations; Springel et al. 2005; Boylan-Kolchin et al. 2009; see also De Lucia \& Blaizot 2007 based on semi-analytic models). Similar methods and results are found in Conroy \& Wechsler (2009), Moster et al. (2010), and Trujillo-Gomez et al. (2010).

Guo et al. (2010) have shown that the SF efficiency in most recent hydrodynamical, cosmological galaxy formation simulations is higher than that predicted from the $M_{\text {star }}-M_{\text {halo }}$ relation. In particular, as shown in Figure 5, the stellar masses of DG1 and DG2 are about an order of magnitude larger than those inferred from the relation.

However, since the SDSS-DR7 only covers the stellar mass range from $10^{8.3}$ to $10^{11.8}$, the $M_{\text {star }}-M_{\text {halo }}$ relation outside this range was extrapolated assuming a constant slope as indicated by the dashed line as shown in Figure 5 (see Guo et al. 2010). The stellar masses of DG1 and DG2 fall within the lower extrapolated region. Here, we make a more direct comparison between the stellar mass to halo mass ratio between simulated and observed galaxies. To this end, we compare DG1 and DG2 with nearby low-mass galaxies from van den Bosch et al. (2001), Stark et al. (2009) and the Local Group as well as the seven dwarfs from THINGS (Oh et al. 2011). 
For the halo mass of the THINGS dwarf galaxies, we estimate $M_{200}$ as follows:

$$
\begin{aligned}
M_{200}\left[M_{\odot}\right] & =200 \times \frac{3 H_{0}^{2}}{8 \pi G} \times \frac{4 \pi R_{200}^{3}}{3} \\
& \simeq 100 \times \frac{H_{0}^{2}}{G} \times\left(\frac{V_{200}}{10 H_{0}}\right)^{3} \\
& \simeq 3.29 \times 10^{5} \times V_{200}^{3},
\end{aligned}
$$

where $H_{0}$ is the Hubble constant $\left(70.6 \mathrm{~km} \mathrm{~s}^{-1} \mathrm{Mpc}^{-1}\right.$; Suyu et al. 2010), $G$ is the gravitational constant $(4.3 \times$ $10^{-3} \mathrm{pc} M_{\odot}^{-1} \mathrm{~km}^{2} \mathrm{~s}^{-2}$ ), and $V_{200}$ in $\mathrm{km} \mathrm{s}^{-1}$ is the rotation velocity at radius $R_{200}$ as given in Equation (1). However, the NFW halo model fails to fit the DM rotation curves of the THINGS dwarf galaxies, giving negative (or close to zero) $c$ values $(\mathrm{Oh}$ et al. 2011). To circumvent the unphysical fits, we instead fit the NFW model to the rotation curves with only $V_{200}$ as a free parameter after fixing $c$ to 5 which is lower than typical values (e.g., 8-9; McGaugh et al. 2003) predicted from $\Lambda$ CDM cosmology. The fitted $V_{200}$ values of some galaxies are larger than their measured maximum rotation velocities. This is because the rotation curves are still rising at the last measured points. Moreover, as a larger $c$ value induces a smaller $V_{200}$ and hence lower halo mass, our choice of a low $c$ will provide a robust upper limit for our derived halo mass, as indicated by the arrows in Figure 5. As shown in Figure 5, despite the uncertainties remaining in these estimates, the stellar masses of DG1 and DG2 at their given halo masses are consistent with those of real galaxies. Both the real galaxies and the simulations deviate from the extrapolated line from the $M_{\text {star }}-M_{\text {halo }}$ relation in Guo et al. (2010) at low halo masses. However, as discussed in Trujillo-Gomez et al. (2010), uncertainties still remain for dwarfs in the sense that the observational data suffer from small number statistics and the results of abundance matching are incomplete in the low-luminosity tail of the luminosity function.

\subsection{The Rotation Curve Shape}

The rotation curve reflects the total potential (DM + baryons) of the galaxy and thus it is directly related to the radial matter distribution in the galaxy (and vice versa). Consequently, the cusp-like DM distributions in the CDM halos impose a unique shape on the rotation curves, which steeply rise at the inner regions. Therefore, a relative comparison of galaxy rotation curves between the simulations and observations can serve as a useful constraint for testing the $\Lambda \mathrm{CDM}$ simulations.

In this context, we compare the rotation curves of DG1 and DG2 with those of the THINGS dwarf galaxies. In order to accentuate their inner shapes, we scale the rotation curves of both the simulations and the THINGS dwarf galaxies with respect to the velocity $V_{0.3}$ at the radius $R_{0.3}$ where the $\log$ arithmic slope of the curve is $d \log V / d \log R=0.3$ (Hayashi $\&$ Navarro 2006). At the scaling radius $R_{0.3}$, the rotation curves of both simulations and the observations are well resolved, which allows any differences between them to show up.

The scaled rotation curves, with the kinematic contribution of baryons subtracted, are shown in the left panel of Figure 6 . We overplot the scaled rotation curves of NFW CDM halos (DM-only) with different maximum rotation velocities ranging from 10 to $350 \mathrm{~km} \mathrm{~s}^{-1}$. We choose $c$ values of $\sim 9$ and $\sim 8$ for dwarf and disk galaxies, respectively, which in turn provide $V_{200}$ values ranging from $\sim 10$ to $\sim 90 \mathrm{~km} \mathrm{~s}^{-1}$, and $\sim 100$

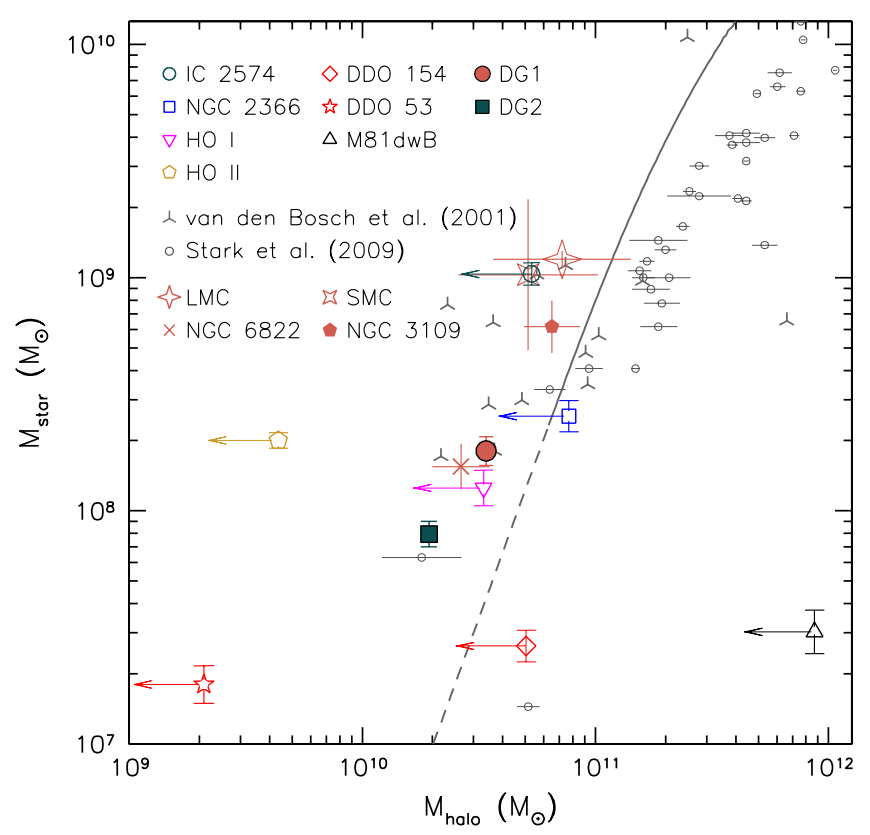

Figure 5. $M_{\text {star }}-M_{\text {halo }}$ relation of DG1, DG2, and the THINGS dwarf galaxies (Oh et al. 2011) as well as galaxies from the literature (van den Bosch et al. 2001; Stark et al. 2009). The Local Group galaxies, LMC (Mastropietro et al. 2005; Guo et al. 2010), SMC (Stanimirović et al. 2004; Guo et al. 2010), NGC 6822 (Valenzuela et al. 2007), and NGC 3109 (Valenzuela et al. 2007) are also overplotted. The error bars for $M_{\text {halo }}$ and $M_{\text {star }}$ of the LMC and SMC are computed based on the different estimates given by Guo et al. (2010) and the other two papers (i.e., Mastropietro et al. 2005; Stanimirović et al. 2004). The error bars for NGC 6822 and NGC 3109 come from the different mass models that Valenzuela et al. (2007) considered for these galaxies to reproduce their kinematical and photometrical properties assuming that they are hosted in CDM halos. The solid curve is from abundance matching by combining the stellar mass function from the SDSS-DR7 with the halo/subhalo mass function from the Millennium and Millennium-II simulations (Guo et al. 2010). As described in Guo et al. (2010), the relation below the stellar mass $10^{8.3}$ was extrapolated assuming a constant slope as indicated by the dashed line. See Section 4.1 for more details.

(A color version of this figure is available in the online journal.)

to $\sim 350 \mathrm{~km} \mathrm{~s}^{-1}$, respectively. Considering that the rotation velocities of DG1, DG2, and the THINGS dwarf galaxies at the outermost measured radii are all less than $\sim 80 \mathrm{~km} \mathrm{~s}^{-1}$, the CDM rotation curve with $V_{200} \sim 90 \mathrm{~km} \mathrm{~s}^{-1}$ (i.e., small dots in Figure 6) can be regarded as a lower limit to the maximum rotation velocities of the galaxies.

As shown in the left panel of Figure 6, the scaled rotation curves of the THINGS dwarf galaxies are similar to each other, showing a linear increase in the inner regions. The inner shapes of the rotation curves are better described by ISO halo models (dashed lines) than NFW models. This implies that the THINGS dwarf galaxies have core-like DM distributions at their centers (see Oh et al. 2011 for more discussion). Similarly, the scaled rotation curves of DG1 and DG2 are consistent with those of the THINGS dwarf galaxies. They significantly deviate from the CDM rotation curves at the inner regions and, like the THINGS dwarf galaxies, they increase too slowly to match the steep rotation curves of the CDM halos.

\subsection{The Dark Matter Density Profile}

A more direct way to examine the central matter distributions in galaxies is to convert the galaxy rotation curve to the mass density profile. In particular, the measurement of the inner slope of the profile provides a stringent observational constraint on the "cusp/core" problem. With an assumption of a spherical 

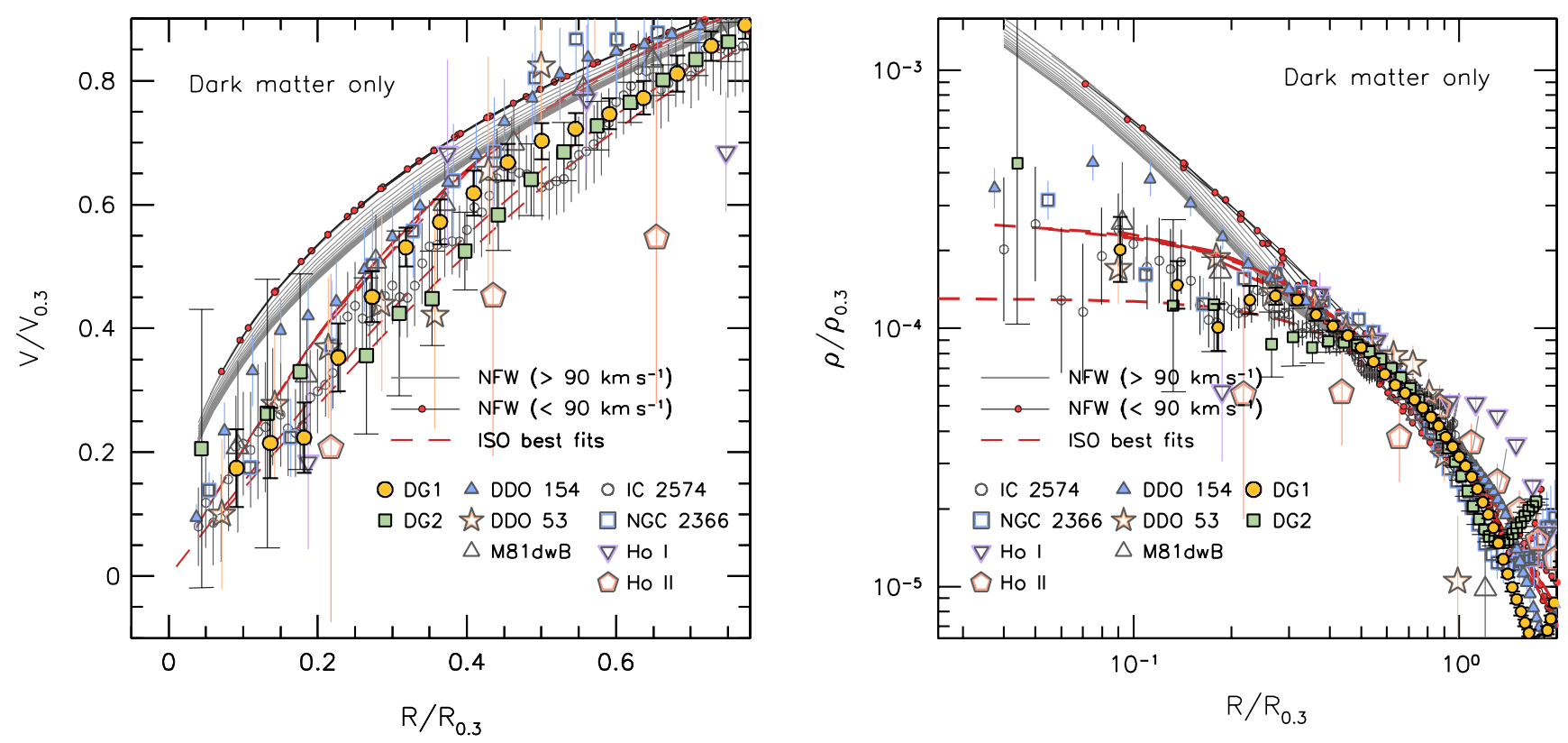

Figure 6. Left: the rotation curve shape of DG1 and DG2 as well as the seven THINGS dwarf galaxies. The DM rotation curves (corrected for baryons as shown in Figure 4) are scaled with respect to the rotation velocity $V_{0.3}$ at $R_{0.3}$ where the $\log$ arithmic slope of the curve is $d \log V / d \log R=0.3$ (Hayashi \& Navarro 2006 ). The small dots indicate the NFW model rotation curves with $V_{200}$ ranging from 10 to $90 \mathrm{~km} \mathrm{~s}^{-1}$. See the text for further details. The best-fitted pseudo-isothermal halo models (denoted as ISO) are also overplotted. See Section 4.2 for more details. Right: the scaled DM density profiles of DG1 and DG2 as well as the seven THINGS dwarf galaxies. The profiles are derived using the scaled DM rotation curves in the left panel. The small dots represent the NFW models $(\alpha \sim-1.0)$ with $V_{200}$ ranging from 10 to $90 \mathrm{~km} \mathrm{~s}^{-1}$. The dashed lines indicate the best-fitted ISO halo models $(\alpha \sim 0.0)$. See Section 4.3 for more details.

(A color version of this figure is available in the online journal.)

mass distribution for the galaxy halo, the galaxy rotation curve $V(R)$ can be converted to the mass density profile $\rho(R)$ by the following formula (see de Blok et al. 2001; Oh et al. 2008, 2011 for more details),

$$
\rho(R)=\frac{1}{4 \pi G}\left[2 \frac{V}{R} \frac{\partial V}{\partial R}+\left(\frac{V}{R}\right)^{2}\right],
$$

where $V$ is the rotation velocity observed at radius $R$ and $G$ is the gravitational constant. Here we do not de-contract the halos since in these galaxies adiabatic contraction does not occur and rather expansion happens as shown in Governato et al. (2010; see also Dutton et al. 2007).

Using Equation (4), we derive the DM density profiles of the THINGS dwarf galaxies, DG1, and DG2 as well as the CDM halos whose rotation curves are shown in the left panel of Figure 6. In addition, we also derive the corresponding mass density profiles of the best-fitted ISO halo models to the THINGS dwarf galaxies. As shown in the right panel of Figure 6, despite the scatter, both DG1 and DG2 have shallower mass density profiles than DM-only simulations. Instead, they are more consistent with the THINGS dwarf galaxies showing near-constant density DM distributions at the centers.

In Figure 7, we compare the derived DM density profiles of DG1 and DG2 with their true full three-dimensional DM density distribution. The inner decrease in the actual DM density profiles of Figure 7 is due to the shape of the potential in the region below the force resolution $(86 \mathrm{pc})$. As shown in Figure 7, for DG1, the observationally derived DM density profile robustly traces the true values but that for DG2 it is found to be on average a factor of three lower than its true value at the central regions. This is mainly due to the lower gas rotation velocity of DG2 as shown in panel (f) of Figure 3, resulting in smaller velocity gradients $\partial V / \partial R$ in Equation (4) and thus smaller densities.
However, considering the uncertainties in deriving the profile, the recovered profile is acceptable to examine the central DM distribution.

We determine the inner density slopes $\alpha$ assuming a power law $\left(\rho \sim r^{\alpha}\right)$ and find them to be $\alpha=-0.31 \pm 0.07$ for DG1 and $\alpha=-0.49 \pm 0.06$ for DG2, respectively. If we re-measure the slope of DG2, excluding the innermost point which has a large error bar, the slope is flatter $(\alpha=-0.27 \pm 0.05)$ as indicated by the long dashed line in the right panel of Figure 7 . These slopes deviate from the steep slope of $\sim-1.0$ from DM-only cosmological simulations. The profiles of both DG1 and DG2 deviate from NFW models beyond about 10 times the force resolution. This tells us that the baryonic feedback processes in dwarf galaxies can affect the DM distribution in such a way that the central cusps predicted from DM-only simulations are flattened, resulting in DM halos characterized by a core, as found in normal dwarf galaxies in the local universe.

\section{CONCLUSIONS}

In this paper, we have compared the DM distribution of the dwarf galaxies from a novel set of $\mathrm{SPH}+\mathrm{N}$-body simulations by Governato et al. (2010) with that of seven THINGS dwarf galaxies to address the "cusp/core" problem in $\Lambda$ CDM. The simulations were performed in a fully cosmological context, and include the effect of baryonic feedback processes, particularly strong gas outflows driven by SNe. Both the simulated and the observed dwarf galaxies have similar kinematic properties and have been analyzed in a homogeneous and consistent manner as described in Oh et al. (2011). The techniques used in deriving DM density profiles were found to provide accurate results when compared with the true underlying profiles, supporting the veracity of the techniques employed by observers. Therefore, this provides a quantitative comparison between the simulations and the observations, and allows us to examine how the baryonic 


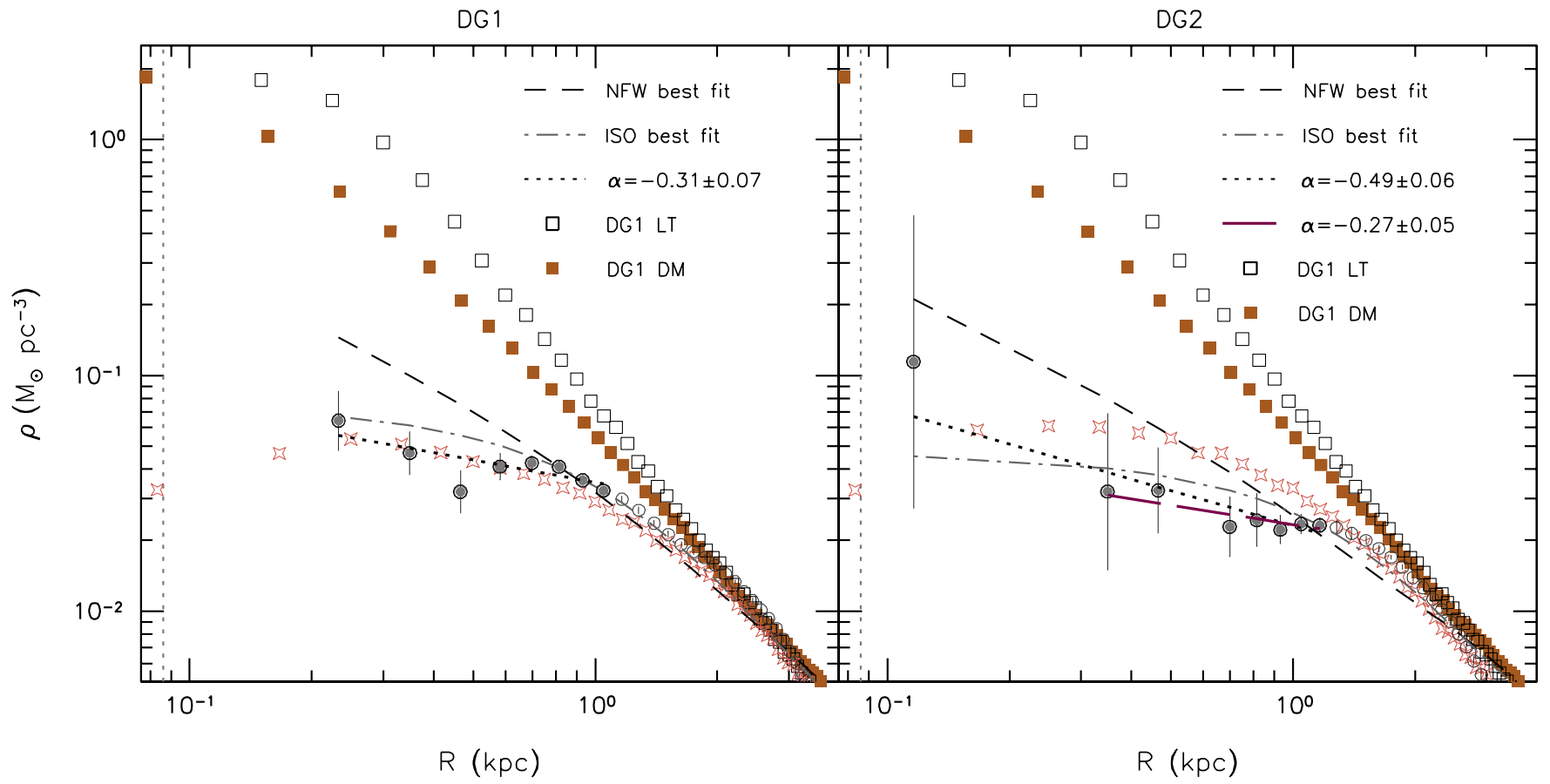

Figure 7. Dark matter density profiles of DG1 (left) and DG2 (right). The circles represent the DM density profiles derived from the DM rotation curves shown in Figure 4. The short dashed and dash-dotted lines indicate the mass density profiles derived using the best-fitted NFW and ISO halo models in Figure 4, respectively. The open and filled rectangles indicate the density profiles of DG1LT and DG1DM, respectively. See Section 2 for more details. The inner slope of the profile is measured by a least-squares fit (dotted lines) to the data points less than $1.0 \mathrm{kpc}$ as indicated by gray dots. In the right panel, the long dashed line indicates a least-squares fit, excluding the innermost point. The measured inner slope $\alpha$ is shown in the panel. The true DM density profiles in the simulations are also overplotted as indicated by open stars. The vertical gray dotted lines indicate the force resolution $(86 \mathrm{pc})$ of the simulations.

(A color version of this figure is available in the online journal.)

feedback processes affect the DM distribution at the centers of dwarf galaxies.

From this, we test the general predictions from $\Lambda \mathrm{CDM}$ simulations: (1) the steep rotation curve inherent in the central cusp and (2) the steep inner slope of $\sim-1.0$ of the DM density profiles. We find that the DM rotation curves of the newly simulated dwarf galaxies rise less steeply at the centers than those from DM-only simulations. Instead, they are more consistent with those of the THINGS dwarf galaxies. In addition, the mean value of the inner density slopes $\alpha$ of the simulated dwarf galaxies is $\simeq-0.4 \pm 0.1$. Compared to the steep slope of $\sim-1.0$ predicted from the previous DM-only simulations (including our simulations run with DM only), these flat slopes are in better agreement with $\alpha=-0.29 \pm 0.07$ found in the seven THINGS dwarf galaxies analyzed by Oh et al. (2011).

In conclusion, the results described in this paper confirm that energy transfer and subsequent gas removal in a clumpy ISM have the net effect of causing the central DM distribution to expand, while at the same time limiting the amount of baryons at the galaxy center. By the present time the DM central profile in galaxies DG1 and DG2 is well approximated by a power law with slope $\alpha$ of $\sim-0.4 \pm 0.1$. These values of $\alpha$ are significantly flatter than in the collisionless control run and are in agreement with those of observed shallow DM profiles in nearby dwarf galaxies.

S.-H.O. acknowledges financial support from the South African Square Kilometre Array Project. F.G. acknowledges support from HST GO-1125, NSF ITR grant PHY-0205413 (also supporting TQ), NSF grant AST-0607819 and NASA ATP NNX08AG84G. The work of W.J.G.d.B. is based upon research supported by the South African Research Chairs Initiative of the
Department of Science and Technology and National Research Foundation. We thank the computer resources and technical support by TERAGRID, ARSC, NAS, and the UW computing center, where the simulations were run.

\section{REFERENCES}

Begeman, K. G. 1989, A\&A, 223, 47

Bell, E. F., \& de Jong, R. S. 2001, ApJ, 550, 212

Benson, A. J., \& Madau, P. 2003, MNRAS, 344, 835

Blais-Ouellette, S., Carignan, C., Amram, P., \& Côté, S. 1999, AJ, 118, 2123

Bolatto, A. D., Simon, J. D., Leroy, A., \& Blitz, L. 2002, ApJ, 565, 238

Boylan-Kolchin, M., Springel, V., White, S. D. M., Jenkins, A., \& Lemson, G. 2009, MNRAS, 398, 1150

Brooks, A. M., Governato, F., Booth, C. M., Willman, B., Gardner, J. P., Wadsley, J., Stinson, G., \& Quinn, T. 2007, ApJ, 655, L17

Bruzual, G., \& Charlot, S. 2003, MNRAS, 344, 1000

Bureau, M., \& Carignan, C. 2002, AJ, 123, 1316

Ceverino, D., \& Klypin, A. 2009, ApJ, 695, 292

Ciotti, L., \& Ostriker, J. P. 2001, ApJ, 551, 131

Conroy, C., \& Wechsler, R. H. 2009, ApJ, 696, 620

Dalcanton, J. J., \& Stilp, A. M. 2010, ApJ, 721, 547

de Blok, W. J. G., \& McGaugh, S. S. 1997, MNRAS, 290, 533

de Blok, W. J. G., McGaugh, S. S., Bosma, A., \& Rubin, V. C. 2001, ApJ, 552, 23

de Blok, W. J. G., Walter, F., Brinks, E., Trachternach, C., Oh, S.-H., \& Kennicutt, R. C. 2008, AJ, 136, 2648

De Lucia, G., \& Blaizot, J. 2007, MNRAS, 375, 2

Diemand, J., Kuhlen, M., Madau, P., Zemp, M., Moore, B., Potter, D., \& Stadel, J. 2008, Nature, 454, 735

Dutton, A. A. 2009, MNRAS, 396, 121

Dutton, A. A., van den Bosch, F. C., Dekel, A., \& Courteau, S. 2007, ApJ, 654, 27

El-Zant, A., Shlosman, I., \& Hoffman, Y. 2002, ApJ, 560, 636

Gentile, G., Burkert, A., Salucci, P., Klein, U., \& Walter, F. 2005, AJ, 634, L145

Ghigna, S., Moore, B., Governato, F., Lake, G., Quinn, T., \& Stadel, J. 2000, ApJ, 544,616

Gnedin, N. Y. 2010, ApJ, 721, L79 
Gnedin, O. Y., \& Zhao, H. 2002, MNRAS, 333, 299

Governato, F., Willman, B., Mayer, L., Brooks, A., Stinson, G., Valenzuela, O., Wadsley, J., \& Quinn, T. 2007, MNRAS, 374, 1479

Governato, F., et al. 2009, MNRAS, 398, 312

Governato, F., et al. 2010, Nature, 463, 203

Guo, Q., White, S. D. M., Li, C., \& Boylan-Kolchin, M. 2010, MNRAS, 404, 1111

Haardt, F., \& Madau, P. 1996, ApJ, 461, 20

Hayashi, E., \& Navarro, J. F. 2006, MNRAS, 373, 1117

Jonsson, P., Groves, B. A., \& Cox, T. J. 2010, MNRAS, 403, 17

Katz, N., \& White, S. D. M. 1993, ApJ, 412, 455

Kennicutt, R. C., Jr., et al. 2003, PASP, 115, 928

Klypin, A., Kravtsov, A. V., Bullok, J. S., \& Primack, J. R. 2001, ApJ, 554, 903

Klypin, A., Kravtsov, A. V., Valenzuela, O., \& Prada, F. 1999, ApJ, 522, 82

Larson, R. B. 1974, MNRAS, 169, 229

Li, C., \& White, S. D. M. 2009, MNRAS, 398, 2177

Mashchenko, S., Couchman, H. M. P., \& Wadsley, J. 2006, Nature, 442, 539

Mashchenko, S., Wadsley, J., \& Couchman, H. M. P. 2008, Science, 319, 174

Mastropietro, C., Moore, B., Mayer, L., Wadsley, J., \& Stadel, J. 2005, MNRAS, 363, 509

McGaugh, S. S., Barker, M. K., \& de Blok, W. J. G. 2003, ApJ, 584, 566

McGaugh, S. S., Schombert, J. M., de Blok, W. J. G., \& Zagursky, M. J. 2010, ApJ, 708, 14

Mo, H. J., \& Mao, S. 2004, MNRAS, 353, 829

Moore, B. 1994, Nature, 370, 629

Moore, B., Ghigna, S., Governato, F., Lake, G., Quinn, T., Stadel, J., \& Tozzi, P. 1999a, ApJ, 524, 19

Moore, B., Quinn, T., Governato, F., Stadel, J., \& Lake, G. 1999b, MNRAS, 310,1147

Moster, B. P., Somerville, R. S., Maulbetsch, C., van den Bosch, F. C., Macciò, A. V., Naab, T., \& Oser, L. 2010, ApJ, 710, 903

Navarro, J. F., Frenk, C. S., \& White, S. D. M. 1995, MNRAS, 275, 56

Navarro, J. F., Frenk, C. S., \& White, S. D. M. 1996, ApJ, 462, 563

Navarro, J. F., Frenk, C. S., \& White, S. D. M. 1997, ApJ, 490, 493

Navarro, J. F., \& Steinmetz, M. 2000, ApJ, 528, 607

Navarro, J. F., \& White, S. D. M. 1994, MNRAS, 267, 401

Navarro, J. F., et al. 2004, MNRAS, 349, 1039

Navarro, J. F., et al. 2010, MNRAS, 402, 21

Oh, S.-H., de Blok, W. J. G., Brinks, E., Walter, F., \& Kennicutt, R. C., Jr. 2011, AJ, 141, 193

Oh, S.-H., de Blok, W. J. G., Walter, F., Brinks, E., \& Kennicutt, R. C. 2008, AJ, 136,2761
Okamoto, T., Gao, L., \& Theuns, T. 2008, MNRAS, 390, 920

Power, C., Navarro, J. F., Jenkins, A. R., Frenk, C. S., White, S. D. M., Springel, V., Stadel, J., \& Quinn, T. 2002, MNRAS, 338, 14

Quinn, T., Katz, N., \& Efstathiou, G. 1996, MNRAS, 278, L49

Read, J. I., \& Gilmore, G. 2005, MNRAS, 356, 107

Reed, D., Governato, F., Verde, L., Gardner, J., Quinn, T., Stadel, J., Merritt, D., \& Lake, G. 2005, MNRAS, 357, 82

Rhee, G., Valenzuela, O., Klypin, A., Holtzman, J., \& Moorthy, B. 2004, ApJ, 617,1059

Robertson, B. E., \& Kravtsov, A. V. 2008, ApJ, 680, 1083

Sánchez-Janssen, R., Méndez-Abreu, J., \& Aguerri, J. A. L. 2010, MNRAS, 406, L65

Simon, J. D., Bolatto, A. D., Leroy, A., \& Blitz, L. 2003, ApJ, 596, 957

Simon, J. D., \& Geha, M. 2007, ApJ, 670, 313

Spekkens, K., Giovanelli, R., \& Haynes, M. P. 2005, AJ, 129, 2119

Springel, V., et al. 2005, Nature, 435, 629

Springel, V., et al. 2008, MNRAS, 391, 1685

Stadel, J., Potter, D., Moore, B., Diemand, J., Madau, P., Zemp, M., Kuhlen, M., \& Quilis, V. 2009, MNRAS, 398, L21

Stanimirović, S., Staveley-Smith, L., \& Jones, P. A. 2004, ApJ, 604, 176

Stark, D. V., McGaugh, S. S., \& Swaters, R. A. 2009, AJ, 139, 312

Stinson, G., Seth, A., Katz, N., Wadsley, J., Governato, F., \& Quinn, T. 2006, MNRAS, 373, 1074

Stoehr, F., White, S. D. M., Springel, V., Tormen, G., \& Yoshida, N. 2003, MNRAS, 345, 1313

Suyu, S. H., Marshall, P. J., Auger, M. W., Hilbert, S., Blandford, R. D., Koopmans, L. V. E., Fassnacht, C. D., \& Treu, T. 2010, ApJ, 711, 201

Swaters, R. A., Madore, B. F., van den Bosch, F. C., \& Balcells, M. 2003, ApJ, 583,732

Tonini, C., Lapi, A., \& Salucci, P. 2006, ApJ, 649, 591

Trujillo-Gomez, S., Klypin, A., Primack, J., \& Romanowsky, A. J. 2010, arXiv: 1005.1289

Valenzuela, O., Rhee, G., Klypin, A., Governato, F., Stinson, G., Quinn, T., \& Wadsley, J. 2007, ApJ, 657, 773

van den Bosch, F. C., Burkert, A., \& Swaters, R. A. 2001, MNRAS, 326, 1205

van den Bosch, F. C., Robertson, B. E., Dalcanton, J. J., \& de Blok, W. J. G. 2000, AJ, 119, 1579

van der Marel, R. P., \& Franx, M. 1993, ApJ, 407, 525

Verde, L., et al. 2003, ApJS, 148, 195

Wadsley, J. W., Stadel, J., \& Quinn, T. 2004, New Astron., 9, 137

Walter, F., Brinks, E., de Blok, W. J. G., Bigiel, F., Kennicutt, R. C., Thornley, M., \& Leroy, A. 2008, AJ, 136, 2563

White, S. D. M., \& Rees, M. J. 1978, MNRAS, 183, 341 